\title{
Effect of bladder dysfunction on development of depression and anxiety in Parkinson's disease
}

\author{
Erdal Benli ${ }^{1}$, Fahriye Feriha Ozer ${ }^{2}$, Nesrin Helvaci Yilmaz ${ }^{3}$, Ozge Arici Duz ${ }^{3}$, Ahmet Yuce ${ }^{4}$, \\ Abdullah Cirakoglu ${ }^{1}$, Tuba Saziye Ozcan ${ }^{5}$ \\ ${ }^{1}$ Department of Urology, Ordu University, Faculty of Medicine, Ordu, Turkey; \\ ${ }^{2}$ Department of Neurology, Koç University Faculty of Medicine, Istanbul, Turkey; \\ ${ }^{3}$ Department of Neurology, Istanbul Medipol University, Faculty of Medicine, Istanbul, Turkey, \\ ${ }^{4}$ Department of Urology, Darende Hulusi Efendi State Hospital, Malatya, Turkey; \\ ${ }^{5}$ Department of Neurology, Sancaktepe Sehit Prof. Dr. Ilhan Varank Training and Research Hospital, Istanbul, Turkey.
}

\begin{abstract}
Summary $\quad$ Objectives: Parkinson's disease (PD) often presents with movement disorder. However, besides motor complaints, there are many complaints such as anxiety, depression, urinary complaints and constipation. The aim of this study was to investigate whether neurogenic lower urinary dysfunction (NLUD), which is frequently seen in PD, has an effect on the development of anxiety and depression in these patients.

Materials and methods: The study included 32 males (66.6\%) and 16 females (33.3\%); in total 48 subjects were registered. For the diagnosis and severity of PD, the UK Parkinson's Disease Society Brain Bank Criteria, Unified Parkinson's Disease Rating Scale (UPDRS) and the Hoehn-Yahr scale were used. Urological evaluation was performed using history, physical examination, laboratory tests and standard forms such as IPSS and OAB-V8.

Results: There was no difference between the genders in terms of duration, severity and NLUD ( $p>0.05)$. The incidence of anxiety and depression in PD patients was $62.8 \%$ and $72.1 \%$, respectively. The prevalence of NLUD was $67.4 \%$ and depression and anxiety was found to increase (1.06 and 1.28 times, respectively) in relation to NLUD. In particular, there was a relationship between storage lower urinary tract symptoms and anxiety and depression development $(p<0.05)$.

Conclusions: As expected, it was found that the incidence of NLUD, anxiety and depression was increased in PD. In addition, NLUD was found to be a risk factor for the development of anxiety and depression. Therefore, it is concluded that NLUD, which can potentially cause important complications, as well as motor complaints, should be closely monitored and treated in $P D$ patients.
\end{abstract}

KEY WORDS: Bladder Dysfunction; Anxiety; Depression; Parkinson's disease.

Submitted 13 May 2021; Accepted 25 June 2021

\section{INTRODUCTION}

Idiopathic Parkinson's disease (PD) is a common movement disorder characterized by damage to dopaminergic neurons in the substantia nigra. It is the second most common neurodegenerative disease after Alzheimer's disease (1). Although the most prominent symptoms of PD are motor disorders such as muscle rigidity, bradykinesia, tremor, many nonmotor symptoms (NMS) may occur in these patients such as sleep disturbances, anxiety, depression, sexual and urinary complaints $(2,3)$. NMSs are known to be associated with deterioration of quality of life or the development of major complications such as head trauma and hip fractures.

Neurogenic lower urinary dysfunction (NLUD) is a common disorder in PD patients that seriously affects the patient's daily work (4). In particular, frequent urination, urgency, and incontinence seriously affect the quality of life. It could lead the patient to stay home to get close to the toilet and away from people.

It is known that psychiatric problems such as anxiety and depression are more common in Parkinson's patients than their healthy peers. The exact cause of psychiatric problems is not known. However, refusal of treatment, deterioration of quality of life, and suicidal thinking can lead to important problems. In our opinion, NLUD, which causes significant problems even in healthy people, may be a cause for the development of anxiety and depression in Parkinson's patients. The aim of this study was to investigate whether there is a relationship between NLUD and psychological problems such as anxiety and depression in PD.

\section{Materials AND METHOdS}

This study was planned jointly by the neurology and urology clinics of Ordu University Medical Faculty.

The files of the patients who were admitted to the movement disorder clinic between 2017 and 2019 with the diagnosis of PD and underwent urological evaluation were evaluated retrospectively. 48 patients who met the study criteria were enrolled in the study. For the diagnosis and severity of Parkinson's disease, the UK Parkinson's Disease Society Brain Bank Criteria, the Unified Parkinson's Disease Rating Scale (UPDRS) and Hoehn-Yahr scale (H\&Y) were used $(5,6)$.

Secondary parkinsonism, psychiatric disease or drug use, history of pelvic radiotherapy or prostate, bladder and gynecological surgery, urinary tract infection, drug use related to prostate or bladder in the last 3 months, 
patients who could not fill in the questionnaire forms by themselves or with the help of their relatives were excluded.

Depression and anxiety levels of the patients were determined by Hamilton Depression (HAMD) and Hamilton Anxiety (HAMA) scales. The HAMD scale consists of 17 questions and the total score is obtained by adding the scores given to each question. The severity of depression is classified according to the total score (0-7 normal, 8-16 moderate depression, > 17 major). The HAMA scale consists of 14 questions and the total score is obtained by adding scores (0-5 points normal, 6-14 medium, > 14 major) (7).

Urological evaluation of the patients was performed by a specialist in this field with history, physical examination and necessary laboratory studies. Urinary complaints of the patients were evaluated using standard questionnaires such as IPSS (International Prostate Symptom Score) and OAB-V8 (overactive bladder-V8). IPSS is an internationally approved form used to describe the patient's lower urinary tract complaints over the past month. The IPSS form evaluates the patient's quality of life (QoL) as well as complaints related to storage and voiding. This form consists of 8 questions, 7 related to urinary system complaints and one related to QoL. Each question is given a score between $0-5$. The total IPSS score in the range of 0-35 is obtained by summing the answers to 7 questions. According to the total IPSS score, LUTS complaints are classified as mild (07), moderate (8-19), and severe (20-35) (8).

The internationally accepted OAB-V8 form was used for storage lower urinary tract symptoms. The presence of at least one of the symptoms such as frequent urination, difficulty in urination, urgency and urinary incontinence associated with urgency was considered as OAB. This form consists of 8 questions and each question is given a score between $0-5$, the total score is obtained by adding these points (9). The study received permission from the local ethics committee (Number: 2020/118).

\section{Statistical analysis}

Statistical analyses were performed using commercial software (Excel Statistics Ver. 20.0). Student-T test was used to compare mean values. The effect of urinary symptoms on anxiety and depression scores was evaluated using binary logistic regression test. The relationship between anxiety and depression scores and QoL and IPSS total scores was evaluated by simple regression and correlation analysis. The $\mathrm{p}$ value of $<0.05$ was used for statistical significance.

\section{ResULtS}

A total of 48 subjects 32 men (66.6\%) and 16 women (33.3\%) were enrolled in the study. Mean age ( \pm std) was $69.84 \pm 7.47$, mean age by gender was $69.22 \pm 7.53$ and $71.64 \pm 7.13$ years for men and women, respectively (not significant difference, $\mathrm{p}=0.36$ ). The distribution of disease duration by gender was $5.29 \pm 3.76$ years for males and $5.64 \pm 3.13$ years for females $(\mathrm{p}=0.78)$. The distribution of UPDRS total score by gender was $25.77 \pm 15.26$ for men and $26.10 \pm 9.32$ for women $(p=0.95)$ (Table 1). There was no difference between the groups in
Table 1.

Demographic distribution of patients.

\begin{tabular}{|ll|c|c|c|}
\hline Parameters & & Mean & Std. dev. & P-value \\
\hline Average age & Men & 69.22 & 7.53 & 0.36 \\
& Women & 71.64 & 7.13 & \\
\hline Disease duration & Men & 5.29 & 3.76 & 0.78 \\
& Women & 5.64 & 3.13 & \\
\hline Age of onset of disease & Men & 63.84 & 8.85 & 0.47 \\
& Women & 66 & 7.62 & \\
\hline UPDR total & Men & 25.77 & 15.26 & 0.95 \\
& Women & 26.10 & 9.32 & \\
\hline
\end{tabular}

terms of disease stage $(\mathrm{p}<0.05)$. HAMD and HAMA mean scores were; $22.95 \pm 9.79(1-42)$ and $8.51 \pm 5.12$ (1-22) respectively.

The prevalence of anxiety and depression in patients with Parkinson's disease was $62.8 \%$ and $72.1 \%$, respectively. The overall mean score of OAB-V8 and IPSS was $12.60 \pm$ $7.18(3-26)$ and $9.58 \pm 6.08$ (1-25), respectively. The distribution of HAMA, HAMD, OAB-V8, IPSS and QoL scores by gender is shown in Table 2 .

Urinary complaints were present in $67.4 \%$ of patients in general and in $68.4 \%$ of males and $63.6 \%$ of females ( $p=$ 0.75 ). Storage lower urinary tract symptoms were found in $61 \%$ in general, $62.8 \%$ in males and $54.5 \%$ in females, respectively $(\mathrm{p}=0.72)$. The prevalence of nocturia was $62.8 \%$ in general, $63.5 \%$ in women and $62.5 \%$ in men (p $=0.94$ ). $83 \%$ of the patients had never been evaluated or questioned in terms of urinary complaints before.

In general, urinary complaints were correlated with anxiety and depression scores ( $p<0.05$ for both). The risk of depression and anxiety increased with the presence of urinary complaints (1.06 and 1.28 times, respectively). In subgroup analyses, especially storage lower urinary tract symptoms were associated with anxiety and depression scores $(\mathrm{p}=0.04)$. This complaint increased the anxiety and depression scores by 1.05 and 1.07 times, respectively. The prevalence of nocturia was $62.8 \%$. Nocturia was found to be associated with both anxiety and depression ( $p<0.05)$. Nocturia increased the risk of anxiety by 1.19 times (Table 3). IPSS total score was correlated with depression and anxiety $(\mathrm{p}=0.03$ and $\mathrm{p}=0.005$, respectively).

Table 2.

Scores of validated questionnairies of asses depression, anxiety and lower urinary tract symptoms.

\begin{tabular}{|ll|c|c|c|}
\hline Parameters & & Mean & Std. dev. & P-value \\
\hline Hamilton anxiety score & Men & 7.5 & 4.42 & 0,06 \\
& Women & 10.72 & 5.44 & \\
\hline Hamilton depression score & Men & 22.15 & 1036 & 0,42 \\
& Women & 25.10 & 8.14 & \\
\hline IPSS total score & Men & 9.78 & 6.24 & 0,717 \\
& Women & 9 & 5.76 & \\
\hline O-life skor & Men & 2.41 & 1.60 & 0,46 \\
& Women & 2 & 1.54 & \\
\hline OAB & Men & 11.94 & 7.02 & 0.61 \\
& Women & 13.45 & 8.75 & \\
\hline
\end{tabular}


Table 3.

Changes in anxiety and depression scores with bladder complaints.

\begin{tabular}{|ll|ccc|ccc|}
\hline Parameters & & Mean & Std. dev. & P-value & Mean & Std. dev. & P-value \\
\hline General urinary complaints & No & 4.55 & 2.50 & 0.01 & 16.11 & 9.41 & 0.01 \\
& Yes & 9.50 & 5.25 & & 25.18 & 8.93 & \\
\hline Storage & No & 6.73 & 5.67 & 0.04 & 18.64 & 10.41 & 0.04 \\
& Yes & 10.41 & 4.96 & & 25.43 & 8.95 & \\
\hline Nocturia & No & 4.69 & 3.06 & $<0.001$ & 18.25 & 10.83 & 0.01 \\
& Yes & 10.42 & 4.90 & & 25.20 & 8.58 & \\
\hline Incontinence & No & 7.33 & 4.85 & 0.02 & 22.73 & 9.45 & 0.84 \\
& Yes & 11.16 & 4.91 & & 23.45 & 11.03 & \\
& & & & & & &
\end{tabular}

Total IPSS score increased the risk of depression and anxiety 1.1 fold. There was a relationship between quality of life score $(\mathrm{QoL})$ and depression and anxiety $(\mathrm{p}=0.03$ and $p=0.03$ ), which increased the risk of anxiety and depression (0.9 and 1.5 times), respectively.

\section{Discussion}

Parkinson 's disease is a multi-systemic disease which is associated with autonomic dysfunction (especially gastrointestinal problems such as constipation and sexual problems such as urinary and erectile dysfunction), anxiety, depression, sleep disturbance as well as motor complaints. The risk of developing at least one NMS in these patients is close to $100 \%(10)$. In our patients, urologic problems, such as urinary complaints, and psychiatric disorders, such as anxiety and depression, were commonly observed.

During daily practice, most PD patients are monitored only for motor complaints and they receive treatment in this regard. In other words, urinary problems are neglected. Our study results support this view. Most of our patients (83\%) had never been evaluated for NLUD before. Patients considered NLUD as the natural consequence of this disease. The results of the study conducted by Gallagher and colleagues also support our conclusions. In this study, NMS incidence and their treatment rates in PD patients were examined. As a result of the study, it was found that one patient had an average of 11 NMS and only 5 of them received treatment (11). Consequently, it was shown that problems other than motor symptoms are frequently neglected in PD patients. Studies have shown that NLUD is a more common nonmotor disorder in PD patients than in the control group (12). The reason for this is not known exactly. Rate of NLUD has been reported in the range of 38-71\% in the literatüre (13). In our study, the incidence of NLUD was $67.4 \%$. OAB which is composed of storage lower urinary tract symptoms such as frequent urination, urgency and incontinence is the most common disorder in this patient group. Uchiyama et al. reported the incidence of $\mathrm{OAB}$ as $64 \%$ (14). In our study, this rate was $61 \%$. Dopaminergic system and autonomic nerve dysfunction in the substantia nigra which is disrupted in PD may be the cause of this pathology (15).

When PD deteriorates, the quality of life is affected due to PD because the patient becomes dependent on other people but also because suffers from urinary dysfunction.
Problems are amplified by a domino effect. For example, nocturia, urgency and fear of urinary incontinence can cause significant problems in the patient's daily life. The daily activities of the patients must be planned in a way that the patient is always close to an accessible toilet and nocturia can cause insomnia and dizziness and in the elderly the risk of falling at night. Thus, the patients start to have problems such as staying at home, loneliness, anger, anxiety, frustration and deterioration of social relations. Consequently the quality of life is severely impaired $(16,17)$. This negative environment may facilitate the development or aggravation of psychiatric problems such as anxiety and depression. In a study on this topic, Starkstein et al. examined the relationship between autonomic symptoms and the development of depression. The authors reported that the presence of 3 or more autonomic symptoms is a strong predictor of depression (18). For this reason, the American Academy of Neurology recommends regular monitoring of NMS in adjunct to motor complaints (19).

It is known that psychiatric problems such as anxiety and depression in PD are more common (7-80\%) than the general population and other chronic neurological diseases such as multiple sclerosis. The exact cause of anxiety and depression in these patients is unknown. It may occur as a part of the neurodegenerative process of PD. NLUD, which seriously deteriorates the quality of life, even in normal people, can trigger or worsen psychiatric problems in these patients.

According to the result of our study, anxiety and depression rates were $76 \%$ and $83 \%$, respectively. In addition, there was a relationship between NLUD and anxiety and depression development. These results were consistent with the literature. In a study on this topic, Engström et al. examined the relationship between NLUD and sadness and joy. The Authors reported that patients with urinary complaints felt unhappy and sad about twice as much as the control group (20). In another study, Irwin et al. examined the relationship between bladder dysfunction and work life, social environment, and emotional well-being. At the end of the study, they reported that NLUD has an effect on social intercourse, productivity in business life and feeling good (21). In another study, Brittian et al. examined the relationship between post-stroke depression and bladder complaints. At the end of the study, they reported that urinary complaints increased the risk of depression twice (15\% vs. 30\%). The Authors suggested that NLUD should be taken seriously in these patients because of the close relationship between depression and suicide (22). In another study, Melvilla et al. reported that the risk of developing depression in women with storage lower urinary tract symptoms increased by 3-4 times (23). In our study, QoL was assessed with a question in the IPSS questionnaire. There was a correlation between QoL and anxiety and depression scores. This result is also important for creating a vicious circle. Deteriorating quality of life triggers psychological problems and psychological problems can lead to more deterioration of quality of life. In one study, the relationship between depression and QoL was investigated. 
The researchers reported that $83 \%$ of depression predicted impaired QoL (24).

In another study by Schwarz et al., the relationship between anxiety and depression and HRQoL (healthrelated quality of life) was examined. In this study, anxiety and depression were reported as the most important risk factors adversely affecting HRQoL (25). When the results of the above study are examined in general, there is an inverse interaction between quality of life and depression and anxiety. Therefore, especially in this age group, because of the close relationship between depression and suicide, the causes of depression in PD patients should be investigated and treated. As seen in the results of our study, NLUD appears to be an important risk factor for the development of anxiety and depression.

In our study, rate of nocturia was found to be $62.8 \%$, and it was also identified as a risk factor for the development of anxiety and depression. Sleep disorders may be the basis of the relationship between nocturia and anxiety and depression.

In other studies, it was found that sleep disorder frequently develops in PD (19). Going to the toilet frequently at night results in a sleep break that may manifest itself with fatigue, dizziness, exhaustion, irritability, and depressive mood. Insomnia, which causes significant problems even in normal individuals, can have more serious consequences in PD patients. For this reason, nocturia in these patients should be noticed in a timely manner and necessary precautions should be taken.

Our study has some limitations. The most important of these is the low number of patients and the retrospective design of the study. In addition, other causes of anxiety and depression were not searched in this study. However, we think that this study is one of the few studies examining the relationship between bladder dysfunction and the development of anxiety and depression in PD.

\section{Conclusions}

In conclusion, NMS such as neurogenic lower urinary dysfunction, anxiety and depression are common in PD patients. However, in daily practice, clinicians who treat these patients often neglect these symptoms because they are more concerned with motor complaints. According to the results of our study, NLUD is a risk factor for the development of anxiety and depression.

These patients, whose quality of life is already deteriorated due to PD, should not be exposed to problems related to NLUD (such as falling, head trauma due to falls, hip fractures, insomnia, anxiety and depression) and the necessary precautions should be followed closely. Patients should not be expected to discuss these complaints, because, as we have seen in our study, these complaints can sometimes be forgotten or considered by patients as a natural part of the disease.

\section{REFEREnCES}

1. Chen JJ. Parkinson's disease: health-related quality of life, economic cost, and implications of early treatment. Am J Manag Care. 2010; 16:87-93.

2. Campos-Sousa RN, Quagliato EM, Almeida KJ, et al. Urinary dys- function with detrusor hyperactivity in women with Parkinson's disease cannot be blamed as a factor of worsening motor performance. Arq Neuropsiquiatr. 2013; 71:591-595.

3. Schrag A, Dodel R, Spottke A, et al. Rate of clinical progression in Parkinson's disease. A prospective study. Mov Disord. 2007; 22:938945.

4. Wong SY, Hong A, Leung J, et al. Lower urinary tract symptoms and depressive symptoms in elderly men. J Affect Disord. 2006; 96:83-88.

5. Fahn S, Elton RL. Unified Parkinson's Disease Rating Scale. In: Fahn S, Marsden CD, Goldsteijn M, Calne DB, Editors. Recent Developments in Parkinson's Disease. Macmillan Healthcare Information. 1987; 2:153-163.

6. Hoehn MM, Yahr MD. Parkinsonism: Onset, progression and mortality. Neurology. 1967; 17:427-442.

7. Maier W, Buller R, Philipp M, Heuser I. The Hamilton Anxiety Scale: reliability, validity and sensitivity to change in anxiety and depressive disorders. J Affect Disord. 1988; 14:61-68.

8. McVary KT, Roehrborn CG, Avins AL, et al. Update on AUA guideline on the management of benign prostatic hyperplasia. J Urol. 2011; 185:1793-1803.

9. Abrams P, Cardozo L, Fall M, et al. Standardisation Sub-committee of the International Continence Society. The standardisation of terminology of lower urinary tract function: report from the Standardisation Sub-committee of the International Continence Society. Neurourology and Urodynamics. 2002; 21:167-178.

10. Moussa M, Papatsoris AG, Abou Chakra M, et al. Erectile dysfunction in common neurological conditions: A narrative review. Arch Ital Urol Androl. 2020; 92:371-385.

11. Gallagher DA, Lees AJ, Schrag A. What are the most important nonmotor symptoms in patients with Parkinson's disease and are we missing them? Mov Disord. 2010; 25:2493-2500.

12. Benli E, Özer FF, Kaya Y, et al. Is there a difference between Parkinson disease patients and a control group in terms of urinary symptoms and quality of life? Turk J Med Sci. 2016; 46:1665-1671.

13. Sakakibara R, Tateno F, Nagao T, et al. Bladder function of patients with Parkinson's disease. Int J Urol. 2014; 21:638-646.

14. Uchiyama T, Sakakibara R, Yamamoto $T$, et al. Urinary dysfunction in early and untreated Parkinson's disease. J Neurol Neurosurg Psychiatry. 2011; 82:1382-1386.

15. Yoshimura N, Kuno S, Chancellor MB, et al. Dopaminergic mechanisms underlying bladder hyperactivity in rats with a unilateral 6-hydroxydopamine (6-OHDA) lesion of the nigrostriatal pathway. Br J Pharmacol. 2003; 139:1425-1432.

16. Campos-Sousa RN, Quagliato EM, Almeida KJ, et al. Urinary dysfunction with detrusor hyperactivity in women with Parkinson's disease cannot be blamed as a factor of worsening motor performance. Arq Neuropsiquiatr. 2013; 71:591-595.

17. Brown JS, Vittinghoff E, Wyman JF, et al. Urinary incontinence: does it increase risk forfalls and fractures? Study of Osteoporotic Fractures Research Group. J Am Geriatr Soc 2000; 48:721-725.

18. Starkstein SE, Preziosi TJ, Forrester AW, Robinson RG. Specificity of affective and autonomic symptoms of depression in Parkinson's disease. J Neurol Neurosurg Psychiatry. 1990; 53:869873

19. Lyons KE, Pahwa R. The impact and management of nonmotor symptoms of Parkinson's disease. Am J Manag Care. 2011; 12:308314 
20. Engström G, Henningsohn L, Steineck G, Leppert J. Self-assessed health, sadness and happiness in relation to the total burden of symptoms from the lower urinary tract. BJU Int. 2005; 95:810-815.

21. Irwin DE, Milsom I, Kopp Z, et al. Impact of overactive bladder symptoms on employment, social interactions and emotional wellbeing in six European countries. BJU Int. 2006; 97:96-100.

22. Brittain KR, Castleden CM. Suicide in patients with stroke. Depression may be caused by symptoms affecting lower urinary tract. BMJ. 1998; 317:1016-1067.
23. Melville JL, Delaney K, Newton K, Katon W. Incontinence severity and major depression in incontinent women. Obstet Gynecol. 2005; 106:585-592.

24. Soh SE, Morris ME, McGinley JL. Determinants of health-related quality of life in Parkinson's disease: a systematic review. Parkinsonism Relat Disord. 2011; 17:1-9.

25. Schwarz J, Odin P, Buhmann C, et al. Depression in Parkinson's disease. J Neurol. 2011; 258:336-338.

\section{Correspondence}

Erdal Benli, MD

Abdullah Cirakoglu, MD

Department of Urology, Ordu University, Faculty of Medicine, Ordu (Turkey)

Fahriye Feriha Ozer, MD

Department of Neurology, Koç University Faculty of Medicine, Istanbul (Turkey)

Nesrin Helvaci Yilmaz, MD

Ozge Arici Duz, MD

Department of Neurology, Istanbul Medipol University, Faculty of Medicine, Istanbul (Turkey)

Ahmet Yuce, MD (Corresponding author)

ahmetyuce7@gmail.com

Department of Urology, Darende Hulusi Efendi State Hospital, Malatya,

Turkey

Tuba Saziye Ozcan, MD

Department of Neurology, Sancaktepe Sehit Prof.Dr. Ilhan Varank Training and Research Hospital, Istanbul (Turkey) 